\title{
A WC9 Star in the LMC
}

\author{
M. Heydari-Malayeri, J. Melnick \\ European Southern Observatory \\ La Silla, Chile
}

\begin{abstract}
We have discovered the only WC9 star in the Large Magellanic Cloud on the basis of observations made at several ESO telescopes, especially NTT. The latest Wolf-Rayet stars of the carbon sequence so far observed in the LMC are WC5-6. The lack of late type WC stars is understood in terms of an abundance effect, according to the models of Maeder. Our observations therefore pose a new observational challenge to the theory of the evolution of massive stars.
\end{abstract}

In the course of a systematic investigation of the HII blobs in the Large Magellanic Cloud, we serendipitously observed a peculiar low excitation, compact HII region which turned out to have several remarkable characteristics. Although this object is catalogued as N82 (Henize, 1956), virtually nothing about it could be found in the literature.

N82 is a relatively very compact HII blob of average radius $1^{\prime \prime} .3$ corresponding to $0.4 \mathrm{pc}$. No internal structure is revealed in the $\mathrm{H} \alpha$ image taken with an excellent seeing of $0^{\prime \prime} .68 \mathrm{FWHM}$, apart from the fact that the object seems somewhat elongated in SE-NE direction. No star is detected in N82.

N82 has a peculiar spectrum. The [NII] $\lambda \lambda 6584,48$ lines are extraordinarily strong (as large as $\mathrm{H} \alpha$ ) and the [O III $] \lambda \lambda 4959,5007$ lines very weak ([O $\mathrm{III}] / \mathrm{H} \beta=1.8$ ). There are also the auroral lines [O III] $\lambda 4363$ and [N II] $\lambda 5755$. These are due to important abundance anomalies in N82. Nitrogen is enriched by a factor of 5 with respect to the average LMC value and oxygen depleted by a factor 2.5 .

The only plausible explanation for these abundance anomalies is that the HII region is ejected by a central W-R star. This is compatible with the fact that the derived electron densities for N82 are unusually large. During the latest stages of massive stars several effects contribute to the change of surface composition. For example, mass loss by stellar winds can progressively reveal nuclearly processed elements, and dredge-up by external convective zones can dilute newly synthesized elements in stellar envelopes. According to models by Maeder (1987), 
a massive star of type $O$ evolves into red supergiant, blue supergiant, nitrogenrich W-R and carbon-rich W-R. At this stage the outer envelopes of the star are thrown out into the interstellar medium. In the case of $\mathrm{N} 82$ the central W-R star should have ejected more than $7 M_{\odot}$.

It is interesting that the derived oxygen and nitrogen abundances correspond very closely to the values predicted by the models of Maeder (1987) for the material shed by massive stars approaching the WC phase. This is an important support for the theory.

The presence of the W-R star embedded in the compact HII region N82 was confirmed by two deep NTT spectra in the blue and red. The presence of several C III lines, especially the $\lambda 4650$ blend and $\lambda 5696$ indicate that we are dealing with a carbon-rich W-R. The absence of C IV lines on the other hand indicate that the star is of a very late type, probably WC9.

Our observations also indicate that the WC9 star is likely to be a member of a binary system, with an early O-type companion. This may resolve the apparent discrepancy with the models which are only valid for single star evolution. According to these models (Maeder, 1990), for low metallicities, only the most massive stars manage to remove their outer layers. In other words, for low $\mathrm{Z}$ the surface composition departs from low $(\mathrm{C}+\mathrm{O}) / \mathrm{He}$ ratios and enters the $\mathrm{WC}$ phase only very late in the helium-burning phase.

In conclusion, $\mathrm{N} 82$ is a high density blob of stellar wind material shed by a massive star, probably in a binary system. The W-R component is the only late type WC star known in the LMC. The present results suggest that the massive stars may play a more important role than has been previously acknowledged in the nitrogen enrichment of the interstellar medium.

For more details see Heydari-Malayeri et al. (1990)

\section{References}

Heydari-Malayeri, M., Melnick, J., Van Drom, E.: 1990, Astron. Astrophys. Letters, in press

Henize,K.G.: 1956, Astrophys. J. suppl. 2, 315

Maeder, A.: 1987, Astron. Astrophys. 173, 247

Maeder, A.: 1990, private communication 